\title{
An ALMA and MagAO Study of the Substellar Companion GQ Lup B*
}

\author{
Ya-Lin Wu ${ }^{1}$, Patrick D. Sheehan ${ }^{1}$, Jared R. Males ${ }^{1}$, Laird M. Close ${ }^{1}$, Katie M. Morzinski ${ }^{1}$, Johanna K. Teske ${ }^{2,5}$, \\ Asher Haug-Baltzell ${ }^{3}$, Nirav Merchant ${ }^{3,4}$, and Eric Lyons ${ }^{3,4}$ \\ ${ }^{1}$ Steward Observatory, University of Arizona, Tucson, AZ 85721, USA; yalinwu @email.arizona.edu \\ ${ }^{2}$ Department of Terrestrial Magnetism, Carnegie Institute of Washington, 5241 Broad Branch Road, NW, Washington, DC 20015, USA \\ ${ }^{3}$ CyVerse, University of Arizona, Tucson, AZ 85721, USA \\ ${ }^{4}$ Bio5 Institute, University of Arizona, Tucson, AZ 85721, USA \\ Received 2016 November 19; revised 2017 January 19; accepted 2017 January 20; published 2017 February 22
}

\begin{abstract}
Multi-wavelength observations provide a complementary view of the formation of young, directly imaged planetmass companions. We report the ALMA $1.3 \mathrm{~mm}$ and Magellan adaptive optics $\mathrm{H} \alpha, i^{\prime}, z^{\prime}$, and $Y_{S}$ observations of the GQ Lup system, a classical T Tauri star with a 10-40 $M_{\text {Jup }}$ substellar companion at $\sim 110$ au projected separation. We estimate the accretion rates for both components from the observed $\mathrm{H} \alpha$ fluxes. In our $\sim 0$ ". 05 resolution ALMA map, we resolve GQ Lup A's disk in the dust continuum, but no signal is found from the companion. The disk is compact, with a radius of $\sim 22$ au, a dust mass of $\sim 6 M_{\oplus}$, an inclination angle of $\sim 56^{\circ}$, and a very flat surface density profile indicative of a radial variation in dust grain sizes. No gaps or inner cavity are found in the disk, so there is unlikely a massive inner companion to scatter GQ Lup B outward. Thus, GQ Lup B might have formed in situ via disk fragmentation or prestellar core collapse. We also show that GQ Lup A's disk is misaligned with its spin axis, and possibly with GQ Lup B's orbit. Our analysis on the tidal truncation radius of GQ Lup A's disk suggests that GQ Lup B's orbit might have a low eccentricity.
\end{abstract}

Key words: accretion, accretion disks - instrumentation: adaptive optics - planets and satellites: individual (GQ Lup B) - stars: individual (GQ Lup) - techniques: interferometric

\section{Introduction}

In recent years, high-contrast imaging surveys have discovered many wide-orbit substellar companions, which are located at tens to hundreds of astronomical units from their host stars and have masses of a few to tens of $M_{\mathrm{Jup}}$. Some of these companions have features indicative of accretion disks, including optical and near-infrared emissions such as $\mathrm{H} \alpha, \mathrm{Br}-\gamma$, and $\mathrm{Pa}-\beta$ (e.g., White \& Ghez 2001; Seifahrt et al. 2007; Schmidt et al. 2008; Bowler et al. 2011, 2014; Patience et al. 2012; Zhou et al. 2014; Lachapelle et al. 2015; Sallum et al. 2015), high dust extinction (e.g., Schmidt et al. 2008; Wu et al. 2015a, 2015b), and infrared excess from dust emission (e.g., Bowler et al. 2011; Bailey et al. 2013; Kraus et al. 2014; Currie et al. 2015). It is expected that disks could be common among young substellar companions because the main formation mechanisms - collapse of prestellar cores, fragmentation of circumstellar disks, and core accretion plus subsequent scattering-can all produce disk-bearing companions. Each mechanism, however, can leave distinct imprints on disk properties. For instance, objects formed by disk fragmentation may have higher disk masses and accretion rates compared to those formed by prestellar core collapse (Stamatellos \& Herczeg 2015). On the other hand, scattering can be destructive to disks (e.g., Reipurth \& Clarke 2001), and unlike stars, low-mass objects are not efficient to accrete new disks from the natal molecular clouds after scattering (Bate 2012). Characterizing disks of substellar companions therefore provides a new avenue for studying wide companions' mass assembly history.

\footnotetext{
* This paper includes data gathered with the $6.5 \mathrm{~m}$ Magellan Clay Telescope at Las Campanas Observatory, Chile.

${ }^{5}$ Carnegie Origins Fellow, joint appointment between Carnegie DTM and Carnegie Observatories.
}

In addition, if gas emission lines such as $\mathrm{CO}$ can be spatially and spectrally resolved, the dynamical mass of the central object can be determined assuming a Keplerian velocity field. Since masses are usually derived by comparing observables to theoretical predictions, this dynamical approach has great potential to calibrate evolutionary models (e.g., Czekala et al. 2015, 2016; MacGregor et al. 2017). Finally, disk masses, sizes, structure, and lifetimes ultimately regulate satellite formation and satellite-disk interaction. Because wide companions are well separated from their host stars, they offer a clear view of the relevant physical processes.

Still, imaging disks around very-low-mass companions remains challenging (e.g., Isella et al. 2014). Simulations have shown that they tend to be compact because they are tidally truncated at $\sim 1 / 3$ of the Hill radius (e.g., Quillen \& Trilling 1998; Ayliffe \& Bate 2009; Shabram \& Boley 2013). For objects in nearby star-forming regions $(\sim 100$ to $150 \mathrm{pc})$, their disks are probably not larger than $\sim 5$ to 30 au in radii, which in turn requires a $<0$ ". 1 resolution to resolve them in (sub)millimeter. With the advent of ALMA, it is now possible to directly image and characterize these disks. Bowler et al. (2015) showed that GSC 6214-210 B has a dust mass of $<0.15 M_{\oplus}$ in its disk. MacGregor et al. (2017) also found that only $<0.04 M_{\oplus}$ of dust is present in the disk around GQ Lup B. Most notably, Kraus et al. (2015) and Caceres et al. (2015) detected FW Tau C's accretion disk in the $1.3 \mathrm{~mm}$ dust continuum and ${ }^{12} \mathrm{CO}(2-1)$ emission, respectively. Kraus et al. (2015) inferred a dust mass of $1-2 M_{\oplus}$, sufficient to form satellites analogous to the Galilean moons.

Here we present the ALMA $1.3 \mathrm{~mm}$ map of the GQ Lup system, a pre-main-sequence star with a 10-40 $M_{\text {Jup }}$ companion at $\sim 110$ au projected separation. Both components have been shown to exhibit accretion signatures (e.g., Batalha et al. 2001; Zhou et al. 2014). With a $\sim 0$ !"05 resolution, we resolve the 
primary star's accretion disk. The companion's disk is, however, not detected. We also present the 0.6-1 $\mu \mathrm{m}$ imaging of the system using the Magellan adaptive optics (MagAO; Close et al. 2012; Kopon et al. 2013; Morzinski et al. 2014), and derive mass accretion rates from $\mathrm{H} \alpha$ intensities.

\section{GQ Lup A and B}

GQ Lup A is a classical T Tauri star (CTTS) with a spectral type of K7eV (Herbig 1962, Herbig 1977) in the Lupus I cloud ( 150 pc; Crawford 2000; Franco 2002). Spectropolarimetric observations suggested that it is $2-5 \mathrm{Myr}$ old, with a photospheric temperature of $4300 \mathrm{~K}$ and a mass of $1.05 M_{\odot}$ (Donati et al. 2012). Photometric monitoring indicated that it has an inclination of $27^{\circ}$ and a rotation period of 8.45 days (Broeg et al. 2007). It is one of the most studied CTTSs due to many features indicative of active accretion onto the star from a circumstellar disk, such as brightness variations (e.g., Friedrich \& Schöffel 1971; Appenzeller et al. 1978; Broeg et al. 2007; Donati et al. 2012), inverse P-Cygni profiles (e.g., Appenzeller et al. 1978; Bertout et al. 1982; Batalha et al. 2001), optical veiling (e.g., Batalha et al. 2001; Seperuelo Duarte et al. 2008), very intense magnetic field (e.g., Donati et al. 2012; JohnsKrull et al. 2013), and strong excess from near- to far-infrared (e.g., Mendoza 1968; Hughes et al. 1994; Kessler-Silacci et al. 2006; McClure et al. 2012; Morales et al. 2012). The accretion disk was first imaged at $1.3 \mathrm{~mm}$ dust continuum emission by Dai et al. (2010) using the Submillimeter Array. The disk is compact, low-mass, and possibly tidally truncated. McClure et al. (2012) presented far-infrared spectra taken with Herschel PACS and proposed that the $63 \mu \mathrm{m}$ excess may come from crystalline water ice in the outer disk. Recently, MacGregor et al. (2017) presented the ALMA $870 \mu \mathrm{m}$ and CO (3-2) imaging of the disk and derived a gas-to-dust ratio well below typical ratios in the interstellar medium (ISM).

The substellar companion GQ Lup B was discovered by Neuhäuser et al. (2005), and its common proper motion was soon confirmed (Mugrauer \& Neuhäuser 2005; Janson et al. 2006; Neuhäuser et al. 2008). Mass estimates are very model-dependent, but most studies overlap in the range of $\sim 10$ to $40 M_{\text {Jup }}$ (see Table 1 of Lavigne et al. 2009 for published results). Like the primary star, GQ Lup B is believed to harbor an accretion disk. Lines of evidence include red $K^{\prime}-L^{\prime}$ compared to young free-floating objects (Kraus et al. 2014), the $1.28 \mu \mathrm{m} \mathrm{Pa}-\beta$ emission line (Seifahrt et al. 2007; but also see McElwain et al. 2007 and Lavigne et al. 2009), and overluminosity in the Hubble Space Telescope (HST) F606W flux (Marois et al. 2007). The $\mathrm{H} \alpha$ emission, along with optical continuum excess, were detected by Zhou et al. (2014). A relatively high accretion rate $\dot{M} \sim 10^{-9.3} M_{\odot} \mathrm{yr}^{-1}$ was derived by modeling the continuum excess as a hot hydrogen slab (Zhou et al. 2014). The dust mass in the disk was shown to be $<0.04 M_{\oplus}$ (MacGregor et al. 2017).

Recently, Schwarz et al. (2016) measured the spin and the barycentric radial velocity (RV) of GQ Lup B using highdispersion spectroscopy. They showed that compared to the giant planet $\beta$ Pic b, GQ Lup B spins slowly because it is still contracting and gaining angular momentum from its disk. Their new RV estimate, together with the astrometric monitoring in Ginski et al. (2014), has provided constraints on its orbit. Schwarz et al. (2016) also detected $\mathrm{CO}$ and $\mathrm{H}_{2} \mathrm{O}$ in GQ Lup B's atmosphere.

We list the properties of GQ Lup A and B in Table 1.
Table 1

Properties of GQ Lup

\begin{tabular}{|c|c|c|c|}
\hline Parameter & GQ Lup A & GQ Lup B & References \\
\hline Distance (pc) & $\sim 150$ & $\sim 150$ & 1,2 \\
\hline Separation $\left({ }^{\prime \prime}\right)$ & $\ldots$ & $0.721 \pm 0.003$ & 3 \\
\hline $\mathrm{PA}\left({ }^{\circ}\right)$ & $\ldots$ & $277.6 \pm 0.4$ & 3 \\
\hline Age (Myr) & $2-5$ & $2-5$ & 4 \\
\hline $\mathrm{SpT}$ & $\mathrm{K} 7 \mathrm{eV}$ & $\mathrm{L} 1 \pm 1$ & 5,6 \\
\hline$A_{V}$ (mag) & $0.4 \pm 0.2$ & $\ldots$ & 7 \\
\hline $\log \left(L / L_{\odot}\right)$ & $0.0 \pm 0.1$ & $-2.47 \pm 0.28$ & 4,6 \\
\hline$T_{\text {eff }}(\mathrm{K})$ & $4300 \pm 50$ & $2400 \pm 100$ & 4,6 \\
\hline Radius & $1.7 \pm 0.2 R_{\odot}$ & $3.4 \pm 1.1 R_{\mathrm{Jup}}$ & 4,8 \\
\hline Mass & $1.05 \pm 0.07 M_{\odot}$ & $\sim 10-40 M_{\text {Jup }}$ & $4,6,9,10,11,12$ \\
\hline $\log \dot{M}\left(M_{\odot} \mathrm{yr}^{-1}\right)$ & -9 to -7 & -12 to -9 & $3,4,13,14,15$ \\
\hline $\log g$ & $3.7 \pm 0.2$ & $4.0 \pm 0.5$ & 4,6 \\
\hline Inclination $\left(^{\circ}\right)$ & $27 \pm 5$ & $\ldots$ & 16 \\
\hline$v \sin (i)\left(\mathrm{km} \mathrm{s}^{-1}\right)$ & $5 \pm 1$ & $5.3_{-1.0}^{+0.9}$ & 4,17 \\
\hline $\begin{array}{l}\text { Rotation Per- } \\
\quad \text { iod (d) }\end{array}$ & $8.45 \pm 0.20$ & $\ldots$ & 16 \\
\hline
\end{tabular}

References. (1) Crawford (2000), (2) Franco (2002), (3) This work, (4) Donati et al. (2012), (5) Herbig (1977), (6) Lavigne et al. (2009), (7) Batalha et al. (2001), (8) GQ Lup B's radius is derived from the adopted $L$ and $T_{\text {eff }}$, (9) Neuhäuser et al. (2008), (10) Marois et al. (2007), (11) McElwain et al. (2007), (12) Seifahrt et al. (2007), (13) Hügelmeyer et al. (2009), (14) Seperuelo Duarte et al. (2008), (15) Zhou et al. (2014), (16) Broeg et al. (2007), (17) Schwarz et al. (2016).

\section{Methodology}

\subsection{ALMA $1.3 \mathrm{~mm}$}

GQ Lup was observed with ALMA in Cycle 3 on UT 2015, November 1 with the Band 6 receiver and $4112 \mathrm{~m}$ antennas reaching a maximum baseline of $14969.3 \mathrm{~m}$. Three of the four available basebands were configured for continuum observations to search for dust emission, each with 128 $15.625 \mathrm{MHz}$ channels for a total of $2 \mathrm{GHz}$ continuum bandwidth, and centered at 233.0, 246.0, and 248.0 GHz. The final baseband was centered at $230.538 \mathrm{GHz}$ with 3840 $0.122 \mathrm{MHz}$ (Hanning smoothed to a resolution of $0.244 \mathrm{MHz}$, or $0.32 \mathrm{~km} \mathrm{~s}^{-1}$ ) channels to search for ${ }^{12} \mathrm{CO}(2-1)$ emission from our targets. Scans of GQ Lup were interleaved with the phase calibrator QSO J1534-3526. The total on-source time was 11.19 minutes.

The data were reduced in the standard way with the CASA software package, using the water vapor radiometry data and QSO J1534-3526 for gain calibration, QSO J1427-4206 for bandpass calibration, and QSO J1337-1257 for flux calibration. The calibrated data were Fourier inverted and deconvolved from the beam using the MSMFS-CLEAN algorithm (Rau \& Cornwell 2011) with no frequency dependence, CLEAN components that are point sources and 1, 2, 4, and 8 times the size of the beam, and natural weighting for the best sensitivity. We produced a continuum map from all four basebands, excluding channels in the -15 to $15 \mathrm{~km} \mathrm{~s}^{-1}$ range of the $\mathrm{CO}$ baseband to avoid contaminating our map with $\mathrm{CO}$ emission. The final $1.3 \mathrm{~mm}$ continuum map was produced after four iterations of phase-only self-calibration using a model produced from the CLEAN algorithm. We show the $1.3 \mathrm{~mm}$ continuum map in Figure 1. The continuum map has an rms of $39 \mu \mathrm{Jy}$ beam $^{-1}$, with a synthesized beam of 0 ". $054 \times 0$ ". 031 and position angle of $68^{\circ} .7$. 


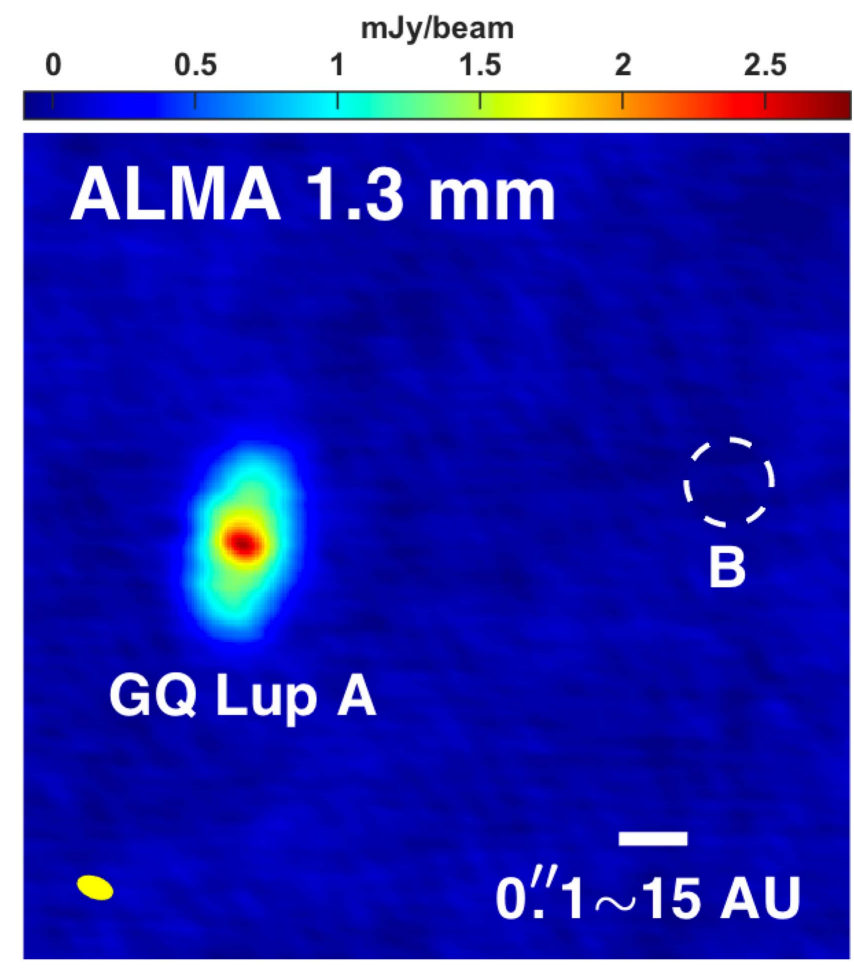

Figure 1. ALMA $1.3 \mathrm{~mm}$ continuum map showing GQ Lup A's accretion disk. We did not detect B's disk (dashed circle). The 0 ." $054 \times 0$.. 031 beam $(8.1$ $\mathrm{au} \times 4.7 \mathrm{au}$ at a distance of $150 \mathrm{pc}$ ) is shown as a yellow oval. North is up and east is left.

\subsection{Sources in the ALMA Image}

In the continuum map, there is a clear detection of a source located at $15^{\mathrm{h}} 49^{\mathrm{m}} 12^{\mathrm{s}} .09,-35^{\circ} 39^{\prime} 05^{\prime \prime}$. 43. Accounting for GQ

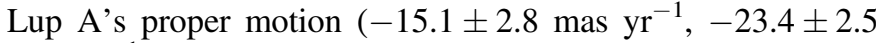
mas $\mathrm{yr}^{-1}$; Zacharias et al. 2010), this is coincident with its reported position at $15^{\mathrm{h}} 49^{\mathrm{m}} 12^{\mathrm{s}} .10,-35^{\circ} 39^{\prime} 05$ !' 12 (J2000). We measured a flux of $27.5 \pm 0.6 \mathrm{mJy}$ for this source.

Because we know the position of GQ Lup B, we can search a smaller region of the image with a lower detection threshold for emission. Noise in our ALMA map is highly Gaussian, so we would expect $68 \%$ of the peaks to be within $1 \sigma$ of zero, $95 \%$ to be within $2 \sigma$, and so on. In a $0 . " 1$ diameter region around the known position of GQ Lup B there are four beams, so we would expect $\sim 0.2$ noise peaks above $2 \sigma$, but $\ll 1$ noise peak above $3 \sigma$, so any peak above $3 \sigma$ is likely real. However, we did not detect any emission from GQ Lup B.

\subsection{GQ Lup B's Disk Mass}

To place an upper limit on the disk mass of GQ Lup B, we inserted fake sources into our image in a 0 ". 1 diameter area around the known position of GQ Lup B, and used our source finding routine to search for them. We varied both the disk size and the flux of the input sources, and for each disk size/flux combination, we calculated the percentage of the fake sources that were recovered in the map. For each disk size, we set the upper limit on disk flux to be the minimum flux for which 99.7\% $(3 \sigma)$ of the input fake sources were detected.

Flux upper limits can be converted into dust mass upper limits by assuming the dust is optically thin and using the

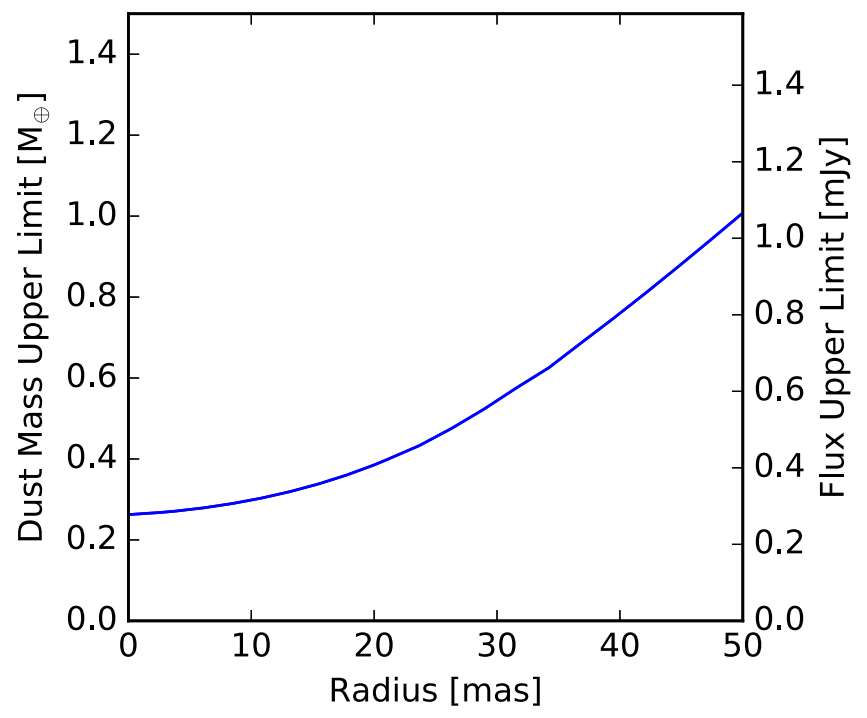

Figure 2. $3 \sigma$ upper limit on the dust mass of the GQ Lup B disk as a function of disk radius. The disk has a flux of $<0.3 \mathrm{mJy}$ corresponding to $<0.25 M_{\oplus}$ of dust if it is a point source, or a flux of $<1.1 \mathrm{mJy}$ corresponding to $<1 M_{\oplus}$ of dust if it reaches $1 / 3$ Hill radius $(\sim 7.5 \mathrm{au})$. We note that the recent $870 \mu \mathrm{m}$ imaging by MacGregor et al. (2017) placed a much stronger constraint on the dust mass, $<0.04 M_{\oplus}$.

standard prescription (Beckwith et al. 1990),

$$
M_{\mathrm{disk}}=\frac{D^{2} F_{\mathrm{disk}}}{\kappa_{\nu} B_{\nu}(T)} .
$$

We used the standard assumption of a characteristic dust temperature of $T=20 \mathrm{~K}$ and a $1.3 \mathrm{~mm}$ dust opacity of $\kappa_{\nu}=2.3 \mathrm{~cm}^{2} \mathrm{~g}^{-1}$. We used a distance of $150 \mathrm{pc}$ to GQ Lup.

In Figure 2, we show a plot of the upper limit on GQ Lup B's disk mass as a function of disk radius. The calculation ran from a point-source disk to a disk with a radius of 50 mas. This radius corresponds to a third of the Hill radius for GQ Lup B, which is expected to be the upper limit on the size of the disk. This calculation also assumed that the projected separation of 0 !'721 is equal to the semimajor axis of the companion's orbit. The Hill radius, and therefore the expected disk radius upper limit, would be larger if the system has a larger separation.

\subsection{GQ Lup A Disk Modeling}

As shown in Figure 1, the disk around the primary star is strongly detected at the $1.3 \mathrm{~mm}$ continuum. We fit the visibility data for GQ Lup A with a series of two disk models to constrain the parameters of the disk.

We modeled the disk with a detailed radiative transfer modeling scheme, using the RADMC-3D code (Dullemond 2012) to produce disk models. Our model included a central protostar with a temperature of $4300 \mathrm{~K}$ and luminosity of $1 L_{\odot}$, consistent with the measured values (see Table 1). It also included a dusty protoplanetary disk, for which we used two different density prescriptions. Model A used the density profile of a flared power-law disk,

$$
\Sigma=\Sigma_{0}\left(\frac{R}{1 \mathrm{au}}\right)^{-\gamma} \text {, and }
$$


Table 2

GQ Lup A Disk Properties

\begin{tabular}{lcc}
\hline \hline Parameter & Model A & Model B \\
\hline Dust Mass $\left(M_{\oplus}\right)$ & $5.9 \pm 1.0$ & $5.5 \pm 0.8$ \\
Total Mass ${ }^{\mathrm{a}}\left(M_{\oplus}\right)$ & $77.2 \pm 8.4$ & $76.8 \pm 8.3$ \\
Inner Radius $(\mathrm{au})$ & $1.5 \pm 0.8$ & $1.7 \pm 1.1$ \\
Radius $(\mathrm{au})$ & $23.8 \pm 1.6$ & $19.5 \pm 1.4$ \\
$h_{0}$ & $0.084 \pm 0.065$ & $0.075 \pm 0.039$ \\
$\gamma$ & $0.10 \pm 0.22$ & $-0.21 \pm 0.20$ \\
$\beta$ & $1.26 \pm 0.19$ & $1.45 \pm 0.25$ \\
Inclination $\left({ }^{\circ}\right)$ & $56.2 \pm 4.8$ & $55.3 \pm 6.0$ \\
PA $\left(^{\circ}\right)$ & $348.8 \pm 4.8$ & $348.6 \pm 5.0$ \\
\hline
\end{tabular}

Note.

${ }^{\text {a }}$ Total mass is calculated by adding our dust mass to the gas mass of $71.3 \pm 8.3 M_{\oplus}$ measured by MacGregor et al. (2017).

$$
\begin{gathered}
\rho=\frac{\Sigma(R)}{\sqrt{2 \pi} h(R)} \exp \left(-\frac{1}{2}\left[\frac{z}{h(R)}\right]^{2}\right), \text { with } \\
h(R)=h_{0}\left(\frac{R}{1 \mathrm{au}}\right)^{\beta} .
\end{gathered}
$$

We allowed the disk mass $\left(M_{\text {disk }}\right)$, inner and outer disk radii ( $R_{\text {in }}$ and $\left.R_{\text {disk }}\right)$, the surface density index $(\gamma)$, scale height index $(\beta)$, and scale height at 1 au $\left(h_{0}\right)$, to vary as free parameters. Model B used the surface density profile of a flared accretion disk with a radial power-law distribution of viscosity (e.g., Lynden-Bell \& Pringle 1974),

$$
\Sigma=\Sigma_{0}\left(\frac{R}{R_{c}}\right)^{-\gamma} \exp \left[-\left(\frac{R}{R_{c}}\right)^{2-\gamma}\right]
$$

The parameters for Model B were the same as those of Model A, with the exception of the critical radius $R_{c}$, beyond which the surface density drops exponentially. This replaced the disk radius, beyond which the density drops to zero in Model A. We also supplied dust opacities to the models. We assumed that dust grains are $70 \%$ astronomical silicate and 30\% graphite (Weingartner \& Draine 2001) following a power-law distribution of dust grain sizes, with a minimum size of $5 \mathrm{~nm}$, a maximum size of $3 \mathrm{~mm}$, and a power-law exponent of -3.5 . This produced a $1.3 \mathrm{~mm}$ opacity of $2.25 \mathrm{~cm}^{2} \mathrm{~g}^{-1}$, in good agreement with the typical value assumed for disk-mass calculations.

We used RADMC-3D to calculate the temperature throughout the density distribution. Following this, we produced synthetic images with raytracing of the protostar model, and Fourier transformed the images to produce model $1.3 \mathrm{~mm}$ visibility profiles. We fit these models directly to the visibility data using the Markov Chain Monte Carlo code emcee (Foreman-Mackey et al. 2013). emcee uses an affine-invariant MCMC ensemble sampler, which employs a series of walkers that step through parameter space and converge on the best fit. We positioned 200 walkers throughout a large region of parameter space and allowed them to move toward regions of lower $\chi^{2}$. We show the best-fit parameters for each model in Table 2, and images, residuals, and visibilities for the best-fit models in Figure 3.

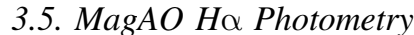

GQ Lup was observed on UT 2015, April 16 in the simultaneous differential imaging (SDI) mode (Close et al. 2014) of the VisAO camera $\left(8^{\prime \prime} \times 8^{\prime \prime}\right.$ field of view, 79 mas plate scale; Close et al. 2013). The rotator was off to facilitate angular differential imaging (ADI; Marois et al. 2006). Weather was photometric with low ground level winds. Seeing varied from 0.7 to 0 !" 8 . AO parameters and exposure time are listed in Table 3. Images in the $\mathrm{H} \alpha \quad(656 \mathrm{~nm} ; \Delta \lambda=6.3 \mathrm{~nm})$ and continuum $(643 \mathrm{~nm}$; $\Delta \lambda=6.1 \mathrm{~nm}$ ) channels were separated, dark subtracted, registered, and centered, and then the median radial profile of each image was subtracted from itself.

After these basic reduction steps, we then proceeded to employ the KLIP algorithm (Soummer et al. 2012) for PSF subtraction with ADI. Our implementation of the ADI+KLIP algorithm allows for the selection of many parameters, including the size of the search region, a minimum rotation requirement, and the number of modes (Males et al. 2014). The following sequence of steps was taken to find a set of signal-to-noise $(\mathrm{S} / \mathrm{N})$ optimizing reduction parameters in an unbiased way.

First, an initial reduction was carried out to locate the companion. This employed a search annulus from 50 to 150 pixels ( 0 ". 4 to 1 !" 2 ), minimum of 2.5 pixels of rotation at the inner edge between the image being reduced and basis images, and modes ranging from 5 to 30. After PSF subtraction, images were de-rotated and the final image was formed as the $5 \sigma$ clipped mean. The final image was then unsharp-masked with a 20-pixel Gaussian kernel, and then smoothed with a 5-pixel Gaussian. Unsharp masking acts as a high-pass filter, removing the stellar halo and the residual low spatial frequency noise remaining after PSF subtraction. Given the low-quality correction, which yielded an FWHM much larger than $\lambda / D$, the PSF was oversampled by the diffraction limited plate scale of the VisAO camera. Gaussian smoothing (low-pass filter) hence smooths pixel-to-pixel noise. The companion was readily seen in the $\mathrm{H} \alpha$ channel with $\mathrm{S} / \mathrm{N} \sim 5$ using eight modes, but no detection was evident in the continuum channel (upper panels of Figure 4).

Next, we injected negative planets (Bonnefoy et al. 2011) with the same search region, using eight modes to form an initial estimate of the planet flux, finding $\Delta \mathrm{H} \alpha \sim 8.7 \mathrm{mag}$ as the planet brightness, which minimized the standard deviation in an aperture with a radius of 1 FWHM at the location of the companion.

It is difficult to estimate uncertainties using the negative planet technique (e.g., Morzinski et al. 2015), and optimizing the reduction parameters on the companion itself risks biasing results due to speckles. To address these issues, we conducted a grid search over the parameters, testing an ensemble of positive fake planets injected at the same separation but at a range of position angles. To conduct this search in a reasonable amount of time, we employed the "Findr" distributed computing (cloudbased) data reduction system (Haug-Baltzell et al. 2016). In all trials, a negative planet with the above estimated brightness was injected at the location of the detection to avoid cross-talk. Planet injection was performed on the dark-subtracted/registered/ centered images, and then the radial profile was subtracted. The parameters tested are given in Table 4, and the KLIP algorithm was applied for each possible combination. The final combined image at each combination was filtered as above. The optimum parameters were determined as those that maximized 

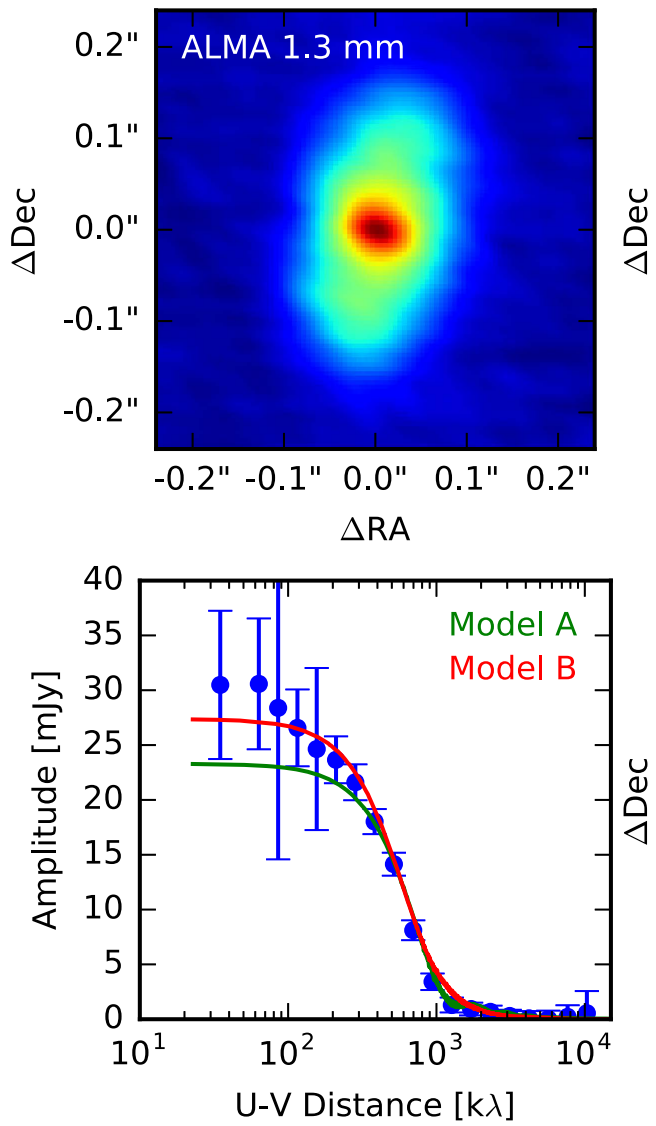
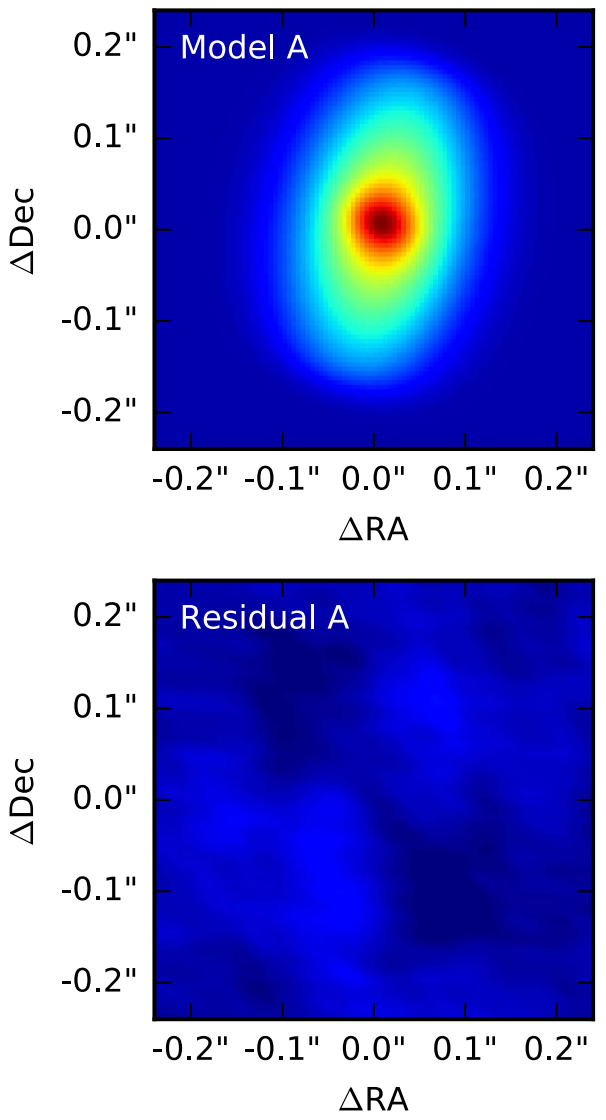
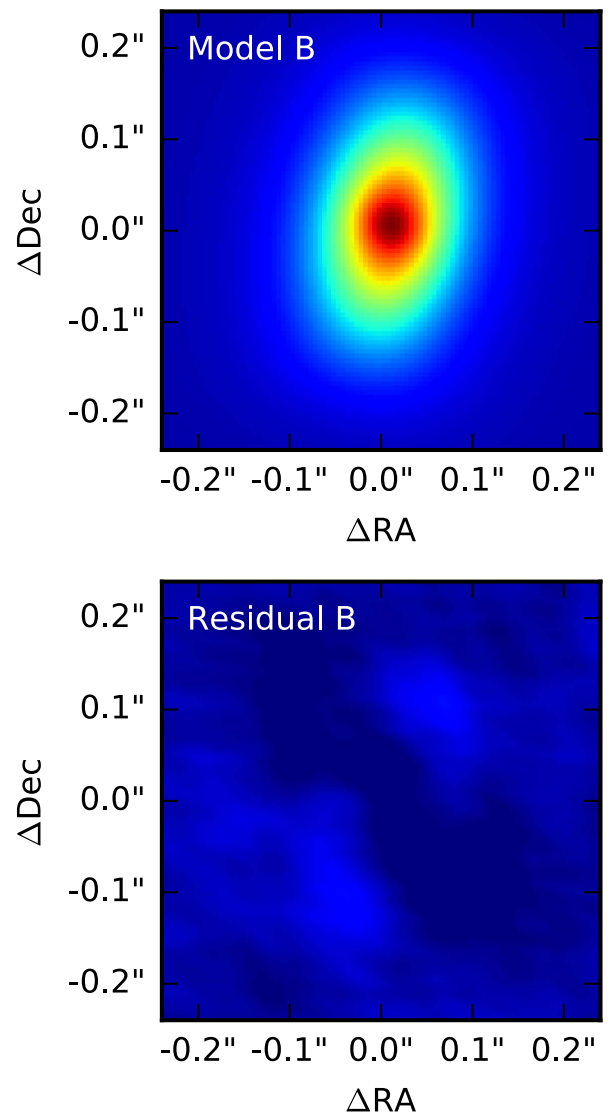

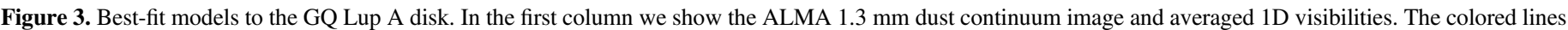
show the best fits for Models A and B. In subsequent columns, we show the best-fit image and residual for each model.

Table 3

MagAO Observations

\begin{tabular}{lllcc}
\hline \hline Filter & AO speed & AO modes & $t_{\text {sat }}$ & $t_{\text {unsat }}$ \\
\hline $643 \mathrm{~nm}$ & $300 \mathrm{~Hz}$ & 120 & $\cdots$ & $25 \mathrm{~s} \times 143$ \\
$656 \mathrm{~nm}(\mathrm{H} \alpha)$ & $300 \mathrm{~Hz}$ & 120 & $\cdots$ & $25 \mathrm{~s} \times 143$ \\
$i^{\prime}$ & $625 \mathrm{~Hz}$ & 120 & $10 \mathrm{~s} \times 20$ & $\cdots$ \\
$z^{\prime}$ & $625 \mathrm{~Hz}$ & 120 & $5 \mathrm{~s} \times 13$ & $0.283 \mathrm{~s} \times 54$ \\
$Y_{S}$ & $625 \mathrm{~Hz}$ & 120 & $\cdots$ & $15 \mathrm{~s} \times 14$ \\
\hline
\end{tabular}

the mean $\mathrm{S} / \mathrm{N}$ on the ensemble of positive fake planets injected at 8.7 mag brightness. The flux of the companion was then determined by comparing its photometry when reduced with those optimum parameters to the positive fake planet results. Uncertainties were estimated from the standard deviation of the results from the fake planets.

At the optimum parameters, GQ Lup B was detected in $\mathrm{H} \alpha$ at $\mathrm{S} / \mathrm{N} \sim 6.3$ with a contrast of $8.60 \pm 0.16 \mathrm{mag}$. For comparison, the $\mathrm{H} \alpha$ contrast in Zhou et al. (2014) is $~ 7.1 \mathrm{mag}$ (Y. Zhou 2016, private communication). The contrast at $643 \mathrm{~nm}$ is $>8.81$ mag ( $3 \sigma$ upper limit). We also found that GQ Lup A's $\mathrm{H} \alpha$ flux is $\sim 0.60$ mag brighter than its $643 \mathrm{~nm}$ continuum due to active accretion.

\subsection{Derivation of Accretion Rates from $\mathrm{H} \alpha$ Fluxes}

We derived the mass accretion rates for GQ Lup A and B following Rigliaco et al. (2012) and Close et al. (2014). In brief, we computed the $\mathrm{H} \alpha$ line luminosity $L_{\mathrm{H} \alpha}$, used it to derive the accretion luminosity $L_{\text {acc }}$, and applied the energy equation $L_{\text {acc }} \propto G M_{\star} \dot{M} / R_{\star}$ to determine $\dot{M}$.

Since we did not observe a standard star at $R$ band, we estimated GQ Lup A's average $R$ brightness to be 11.0 mag by averaging literature fluxes in Schwartz \& Noah (1978), Bertout et al. (1982), Kardopolov \& Filipev (1985), and Covino et al. (1992), with no attempt to homogenize photometric systems. The uncertainty was taken to be 0.7 mag because Broeg et al. (2007) found that GQ Lup A's $R$ flux can change by 1.4 mag over its 8.45-day rotation period. To recover the true $L_{\mathrm{H} \alpha}$, we also have to correct for dust extinction, which is $A_{V} \sim 0.4 \mathrm{mag}$ to the star (Batalha et al. 2001) but unknown to the companion. As a result, our $\dot{M}$ estimate for GQ Lup B should be considered a lower limit.

We thus estimated $L_{\mathrm{H} \alpha} \sim 10^{-2.3}$ to $10^{-1.8} L_{\odot}$ for $\mathrm{A}$, and $\sim 10^{-5.9}$ to $10^{-5.4} L_{\odot}$ for B. Substituting $L_{\mathrm{H} \alpha}$ into the empirical relation, $\log \left(L_{\mathrm{acc}}\right)=2.99+1.49 \times \log \left(L_{\mathrm{H} \alpha}\right)$, in Rigliaco et al. (2012), we found $L_{\text {acc }} \sim 0.3$ to $2.3 L_{\odot}$ for $\mathrm{A}$, and $\sim 1.4 \times 10^{-6}$ to $9.7 \times 10^{-6} L_{\odot}$ for $\mathrm{B}$. The resulting accretion rates for GQ Lup A and B are $\dot{M} \sim 10^{-8}$ to $10^{-7} M_{\odot} \mathrm{yr}^{-1}$, and $\sim 10^{-12}$ to $10^{-11} M_{\odot} \mathrm{yr}^{-1}$, respectively. Our measurement for A is similar to previous results, $\dot{M} \sim 10^{-9}$ to $10^{-7} M_{\odot} \mathrm{yr}^{-1}$ (Seperuelo Duarte et al. 2008; Hügelmeyer et al. 2009; Donati et al. 2012). On the other hand, for the companion we obtained a lower value compared to $10^{-9.3} M_{\odot} \mathrm{yr}^{-1}$ in Zhou et al. (2014). Possible causes include the unknown dust extinction or an inactive period of accretion during the time of our observations. Our $\dot{M}$ estimates and literature values are also shown in Table 1. 

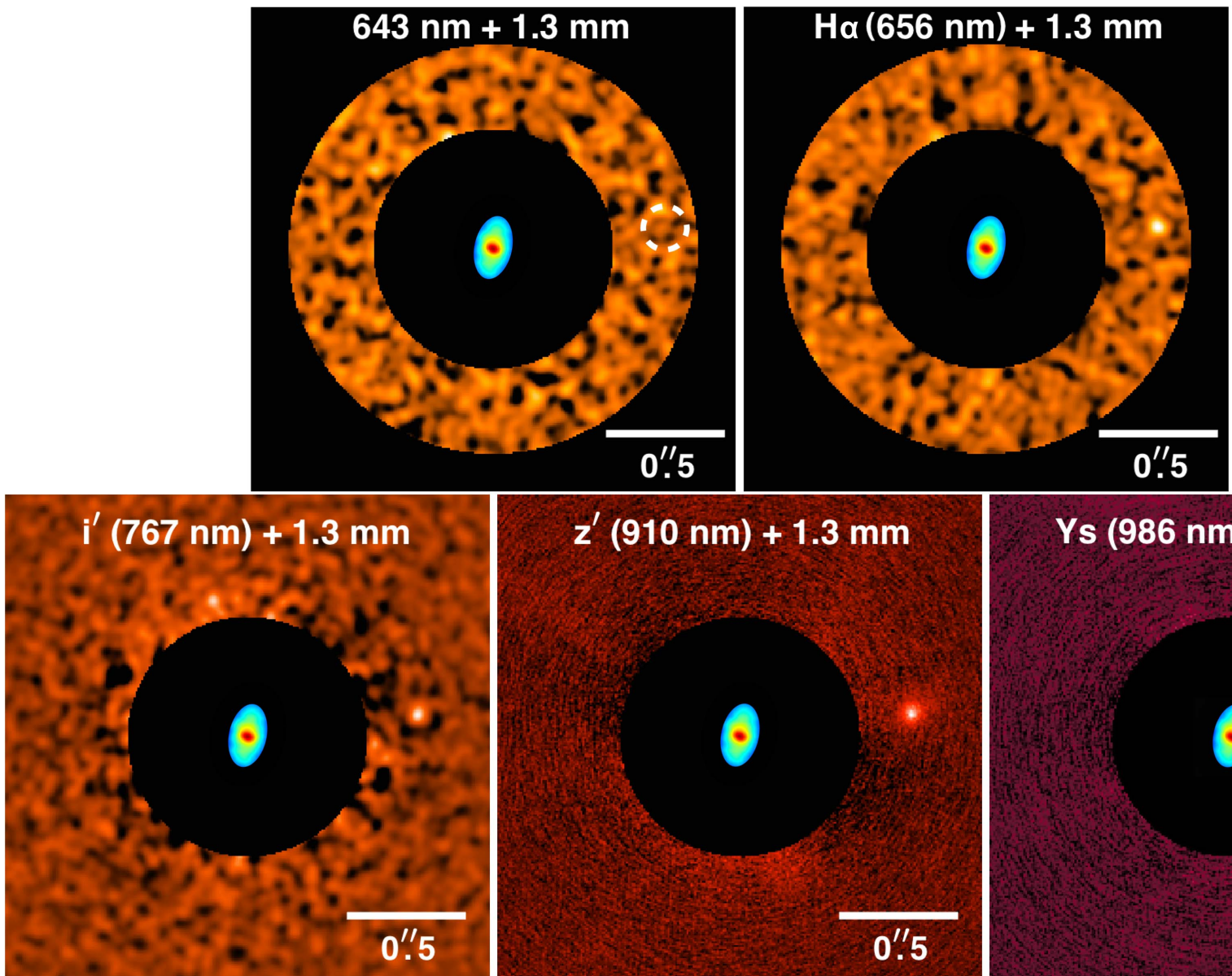

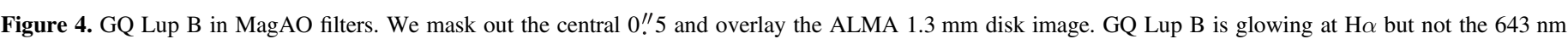
continuum, indicating active accretion. North is up and east is left.

\subsection{MagAO i', $z^{\prime}, Y_{S}$ Photometry}

We observed GQ Lup at broadband filters $i^{\prime}(0.77 \mu \mathrm{m}$, $\Delta \lambda=0.13 \mu \mathrm{m}), \quad z^{\prime} \quad(0.91 \mu \mathrm{m} ; \quad \Delta \lambda=0.12 \mu \mathrm{m}), \quad$ and $\quad Y_{S}$ $(0.98 \mu \mathrm{m} ; \Delta \lambda=0.09 \mu \mathrm{m})$ on UT 2014 April 5. Weather was partially cloudy with $\sim 1$ !" 3 seeing. Data reduction and photometry were carried out with IRAF ${ }^{6}$ (Tody 1986; Tody et al. 1993) and MATLAB. Raw data were dark-subtracted, registered, de-rotated, and median-combined. We then subtracted the median radial profile of the combined image from itself. For $i^{\prime}$, we further filtered out the residual using a 15-pixel Gaussian kernel, and smoothed the resulting high-pass filtered image with a six-pixel Gaussian to better bring out the companion. Our $i^{\prime}, z^{\prime}$, and $Y_{S}$ detections of GQ Lup B are also shown in Figure 4.

We estimated GQ Lup B's fluxes by injecting fake planets. For $z^{\prime}$ and $Y_{S}$, the primary star in the unsaturated data was used to create fake planets. For $i^{\prime}$, since we did not acquire any unsaturated data, we estimated the peak height of the primary star in the saturated data from its beam splitter optical ghost (Males 2016). Then, we flagged the saturated core, assigned the peak value to the center, and fit a two-component Gaussian to create a PSF template. To compensate for flux loss from data

\footnotetext{
6 IRAF is distributed by the National Optical Astronomy Observatories, which are operated by the Association of Universities for Research in Astronomy, Inc., under cooperative agreement with the National Science Foundation.
}

reduction, including filtering, we injected fake planets at the same separation from the star but with different position angles, and repeated the data reduction procedures. We found that throughputs were $>98 \%$ for $z^{\prime}$ and $Y_{S}$, but only $\sim 32 \%$ for $i^{\prime}$ as it involved more aggressive spatial filtering and so higher losses of low spatial frequency flux. Compensating for these flux losses, we derived contrasts of $8.13 \pm 0.23 \mathrm{mag}$, $6.63 \pm 0.05 \mathrm{mag}$, and $6.45 \pm 0.05 \mathrm{mag}$ for $i^{\prime}, z^{\prime}$, and $Y_{S}$, respectively.

To perform absolute photometry, we compared GQ Lup A to the standard star GJ 440. We used an 80-pixel aperture to include most of the flux, and adopted an $8 \%$ photometric uncertainty recommended for absolute photometry with the VisAO camera by Males (2016). Finally, we obtained $i^{\prime}=10.76 \pm 0.08 \mathrm{mag}$, $z^{\prime}=9.77 \pm 0.08 \mathrm{mag}$, and $Y_{S}=9.43 \pm 0.08 \mathrm{mag}$ for GQ Lup $\mathrm{A}$, and $i^{\prime}=18.89 \pm 0.24 \mathrm{mag}, z^{\prime}=16.40 \pm 0.10 \mathrm{mag}$, and $Y_{S}=15.88 \pm 0.10$ mag for GQ Lup B. For comparison, our $z^{\prime}$ flux is similar to the F850LP flux of $16.2 \mathrm{mag}$ in Zhou et al. (2014), but our $i^{\prime}$ flux is $\sim 1$ mag fainter than their F775W flux of 17.8 mag. It is possible that GQ Lup B was in a quiescent accretion state during our observations. Table 5 summarizes our photometric measurements.

\subsection{MagAO Astrometry of $G Q$ Lup $B$}

Following the calibrations in Close et al. (2013) and Males et al. (2014), in our MagAO data, we found that GQ Lup B is 0 ."721 \pm 0 ".003 from its host star, with a position angle of 
Table 4

Parameters of $\mathrm{H} \alpha$ KLIP Reduction

\begin{tabular}{|c|c|c|c|}
\hline Parameter & Grid & Optimum & Notes \\
\hline Minimum radius of region (pixel) & $40-80$, steps of 10 & 50 & $\cdots$ \\
\hline Maximum radius of region (pixel) & $110-140$, steps of 10 & 110 & $\cdots$ \\
\hline Minimum rotation (pixel) & $0.0,0.25,0.5,1.0,2.0$ & 1.0 & $\cdots$ \\
\hline Number of modes & $2-20$, steps of 2 & 6 & $\cdots$ \\
\hline Fake planet PA & $7.15-357.15$, steps of 10 & $\cdots$ & 33 total, skipped $\pm 10^{\circ}$ from planet \\
\hline Fake planet contrast & $1.65,3.30,4.95 \times 10^{-4}$ & $\cdots$ & $\pm 50 \%$ from negative planet result \\
\hline
\end{tabular}

Table 5

MagAO Photometry

\begin{tabular}{|c|c|c|c|}
\hline Contrast/Filter & GQ Lup A & & GQ Lup B \\
\hline$\Delta 643 \mathrm{~nm}^{\mathrm{a}}$ & & $>8.81$ & \\
\hline$\Delta \mathrm{H} \alpha$ & & $8.60 \pm 0.16$ & \\
\hline$i^{\prime}$ & $10.76 \pm 0.08$ & & $18.89 \pm 0.24$ \\
\hline$z^{\prime}$ & $9.77 \pm 0.08$ & & $16.40 \pm 0.10$ \\
\hline$Y_{S}$ & $9.43 \pm 0.08$ & & $15.88 \pm 0.10$ \\
\hline
\end{tabular}

Note.

a $3 \sigma$ upper limit.

$277^{\circ} .6 \pm 0.4$. Figure 5 shows the astrometric monitoring in the last $\sim 20$ years, and GQ Lup B's orbital motion is evident. Our results are consistent with the trends derived by Ginski et al.

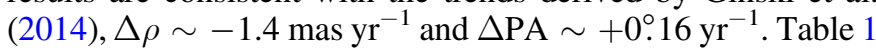
also lists our astrometric measurements.

\section{Results}

\subsection{GQ Lup A's Disk}

We list the best-fit parameters for our modeling of the GQ Lup A disk in Table 2, and show our best-fit models in Figure 3. Both models match the data well, and produce similar best-fit parameter values. We find that GQ Lup A's disk has a radius of $\sim 22 \mathrm{au}$, an inclination angle of $\sim 56^{\circ}$, and a position angle of $\sim 349^{\circ}$. The mass in dust in the disk is $\sim 6 M_{\oplus}$, lower than $\sim 9.5 M_{\oplus}$ found by Dai et al. (2010) and $\sim 15 M_{\oplus}$ found by MacGregor et al. (2017). This difference in dust mass likely comes from the adopted temperature profiles in the disk. In this study, we use radiative transfer to calculate the local temperature. Alternatively, if we assume a constant temperature of $20 \mathrm{~K}$ throughout the entire disk and use Equation (1) to calculate the dust mass from the measured flux of $27.5 \mathrm{mJy}$, we obtain a higher value of $\sim 18 M_{\oplus}$.

We find that the disk size we measure $(R \sim 22 \mathrm{au})$ is smaller than the size measured by MacGregor et al. (2017; $R \sim 30$ au from $870 \mu \mathrm{m}$ continuum, and $R \sim 46.5$ au from $\mathrm{CO}$ (3-2) emission). This may be because dust grain growth is expected to occur preferentially in the inner disk, where densities are higher, and radial drift will tend to concentrate large particles at smaller radii. Our $1.3 \mathrm{~mm}$ map is more sensitive to large dust grains than the $870 \mu \mathrm{m}$ map in MacGregor et al. (2017), so we may expect to measure a smaller radius at longer wavelengths.

Our map does not show any structures in the GQ Lup A disk such as holes or gaps, which can be the signposts of additional companions. The best-fit disk models also have very small inner disk radii, of $\sim 1.6 \mathrm{au}$. This is consistent with no inner clearing, because the resolution of our observations does not allow us to well constrain the inner radius below $\sim 4.5$ au. Since there are unlikely any gaps or companions hidden within the disk, GQ Lup B was probably not scattered to its current orbit, but instead formed in situ like binary stars, as we discuss further in Section 5.

Our models show that the surface density profile of the disk can be very flat. The flat profile is similar to some brown dwarf disks in $\rho$ Ophiuchus (Testi et al. 2016), but in contrast to brown dwarf disks in Taurus (Ricci et al. 2014), which have rather steep profiles and smooth edges. As Testi et al. (2016) argued, if dust has a radial variation in the size distribution, assuming uniform dust properties across the disk can result in a shallower profile. This has been confirmed by McClure et al. (2012), who showed that GQ Lup A's disk does have a radial gradient in both the dust composition and grain size, with larger grains in the inner disk and submicron grains in the outer disk. The smaller disk size measured at $1.3 \mathrm{~mm}$ compared to $870 \mu \mathrm{m}$ provides further evidence that larger grains are present in the inner disk.

\subsection{GQ Lup B's Disk Mass}

The disk around GQ Lup B is undetected in our map. As shown in Figure 2, our data put an upper limit on the disk mass for GQ Lup B at $<0.25-1 M_{\oplus}$ from a point source to one-third of the Hill radius, though this is not as strong as the upper limit of $<0.04 M_{\oplus}$ by MacGregor et al. (2017). Unlike another wideorbit substellar companion FW Tau C, which has 1 to $2 M_{\oplus}$ of dust in its disk (Kraus et al. 2015), GQ Lup B's disk appears to have little dust, similar to the dust-depleted disk around GSC 6214-210 B $\left(<0.15 M_{\oplus}\right.$ of dust; Bowler et al. 2015). This may arise from different evolutionary stages: $\mathrm{FW}$ Tau $\mathrm{C}$ is younger ( $\sim 2 \mathrm{Myr})$ and has a more massive accretion disk, while GQ Lup B (2-5 Myr) and GSC 6214-210 B (5-10 Myr) are more evolved and their disks are rather depleted.

\subsection{Orbital Constraints from Disk Size}

GQ Lup B's orbital motion was first detected by Ginski et al. (2014), who showed that the best-fit orbits were eccentric. With new RV measurements, Schwarz et al. (2016) showed that the semimajor axis $a$, eccentricity $e$, and inclination $i$ of GQ Lup B's orbit fall into three groups:

1. $a \sim 100 \mathrm{au}, e \sim 0.15, i \sim 57^{\circ}$,

2. $a<185 \mathrm{au}, 0.2<e<0.75,28^{\circ}<i<63^{\circ}$, and

3. $a>300 \mathrm{au}, e>0.8,52^{\circ}<i<63^{\circ}$.

As was argued by Schwarz et al. (2016), Group 3 is a priori unlikely because its high eccentricity and long orbital period would mean that we are observing GQ Lup B close to periastron. Since the circumstellar disk may be disrupted if the companion goes too close to the star, we can calculate the truncation radius for each of the orbit groups to determine 

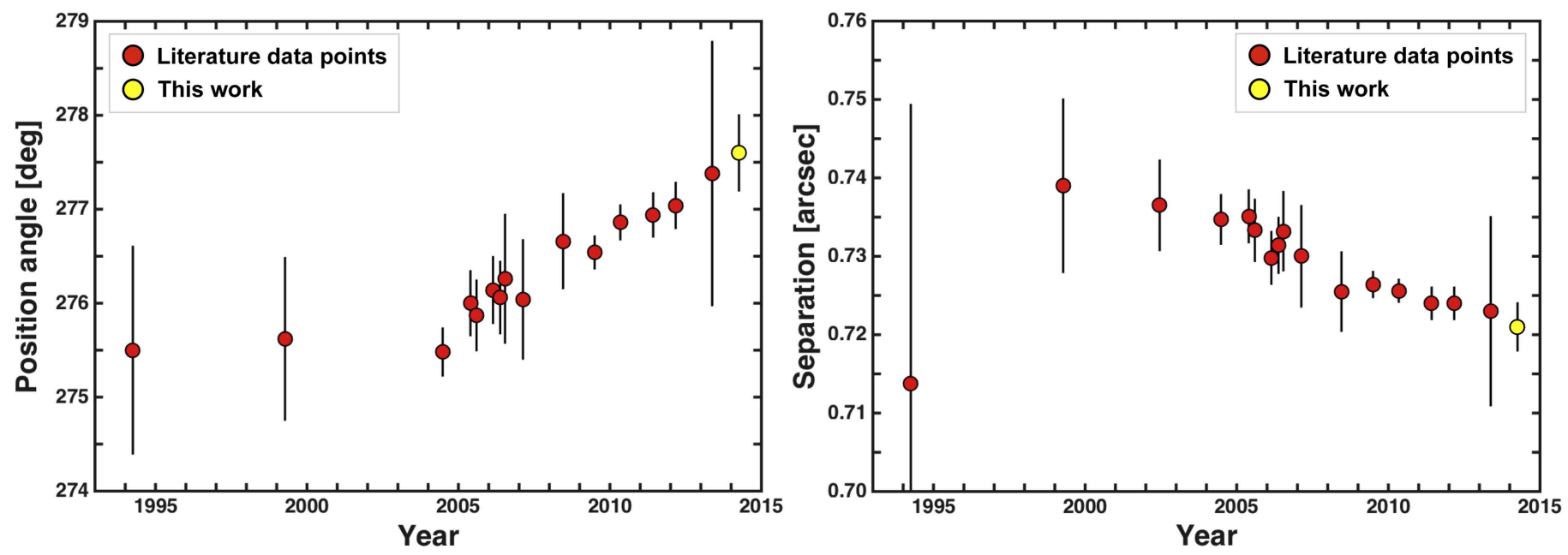

Figure 5. Orbital motion of GQ Lup B over the last two decades. Existing data points are compiled from Janson et al. (2006), Neuhäuser et al. (2005, 2008), Ginski et al. (2014), and Uyama et al. (2017). Figure adapted from Ginski et al. (2014).
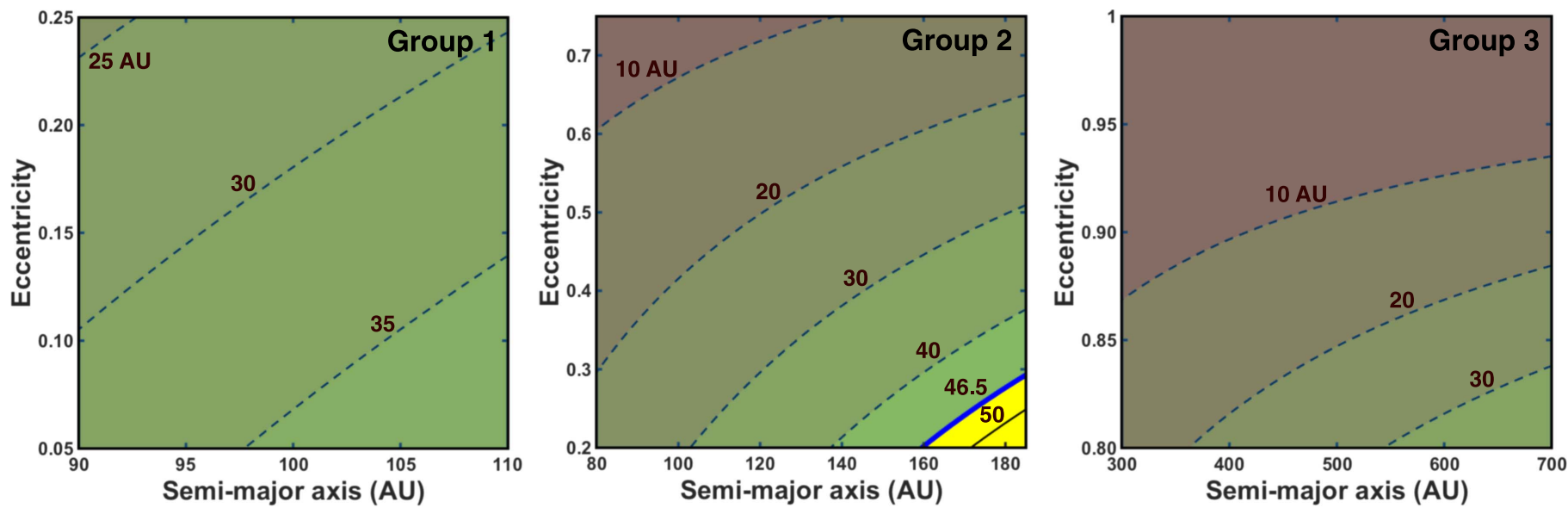

Figure 6. Tidal truncation radius of GQ Lup A's disk as a function of the semimajor axis and eccentricity of GQ Lup B's orbit for the three groups of possible orbits in Schwarz et al. (2016). Contours represent lines of constant truncation radius. Orbits should have a tidal truncation radius greater than $\sim 46.5$ au to be consistent with the measured size of GQ Lup A's gas disk. Therefore, Groups 1 and 3 are probably rejected since their truncation radii are less than 40 au. GQ Lup B's orbit may have $a \sim 170$ au and $e \sim 0.25$, as shown in the yellow region of Group 2 .

whether they are consistent with the size of the GQ Lup A disk. We adopt a disk radius of 46.5 au determined by MacGregor et al. (2017) from CO (3-2) emission, as it more likely represents the full extent of the disk compared with our $1.3 \mathrm{~mm}$ size measurement.

Semi-analytic approximations for the tidal truncation radius of the primary star as a function of orbital parameters find that the disk should be truncated at a radius of

$$
R_{t} \approx 0.36\left[\frac{(1-e)^{1.2} \phi^{2 / 3} \mu^{0.07}}{0.6 \phi^{2 / 3}+\ln \left(1+\phi^{1 / 3}\right)}\right] a,
$$

where $\phi$ is the ratio of the mass of the primary to the mass of the secondary, and $\mu \equiv 1 /(1+\phi)$ (Eggleton 1983; Pichardo et al. 2005). This equation is relatively insensitive to whether the mass of GQ Lup B is closer to $10 M_{\text {Jup }}$ or $40 M_{\text {Jup }}$ (because it is at most $4 \%$ of the primary's mass), but is very sensitive to eccentricity and semimajor axis. We hence arbitrarily use 25 $M_{\text {Jup }}$ for this calculation.

We show the truncation radius as a function of semimajor axis and eccentricity in Figure 6. We find that the truncation radii for Group 1 and Group 3 are all less than 40 au, not compatible with the measured disk size. Most of the parameter space for Group 2 is also excluded; the remaining solutions are those with $a \sim 160-180$ au and $e \sim 0.2-0.3$. As a result, the size of the GQ Lup A disk suggests that GQ Lup B's orbit probably has a low eccentricity. However, it is important to note that this equation may break down if the primary's disk and the secondary disk's orbit are substantially inclined (as may be the case for GQ Lup, see Section 4.4). In this scenario, it is likely that larger disk radii than we conclude here would be acceptable. Indeed, the strong constraints we place here based on this analysis may instead be an indication of a high degree of inclination for the orbit.

\subsection{Geometry of the System}

Since the inclination of GQ Lup A's disk, $\sim 56^{\circ}$, is consistent with orbital solutions in Ginski et al. (2014) and Schwarz et al. (2016), here we investigate if the star's disk and the companion's orbit can possibly be in the same plane.

In Figure 7, we plot the three best-fit orbits in Ginski et al. (2014; see their Table 4 for orbital parameters) as well as the GQ Lup A disk in two viewing angles, one along the line of sight and the other with the disk viewed edge-on. We also show one 

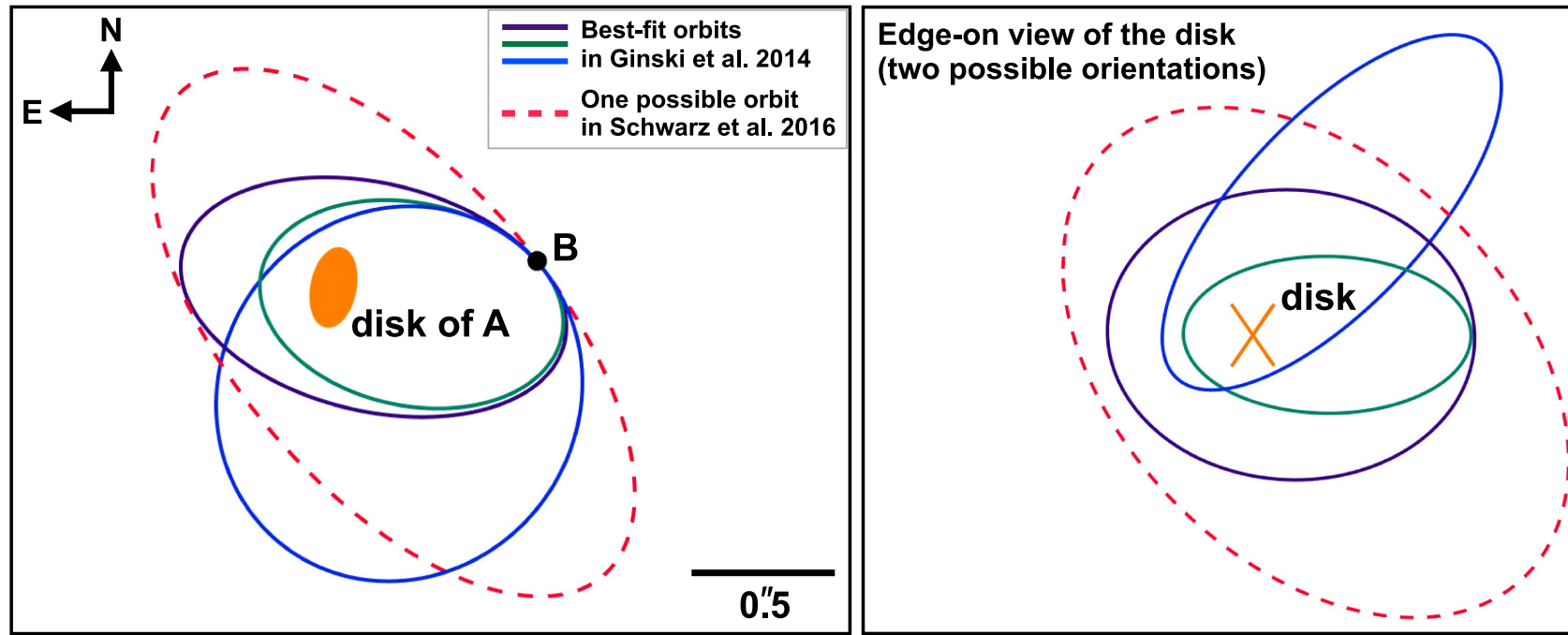

Figure 7. Disk of GQ Lup A and orbital solutions of GQ Lup B. Left: when the system is projected on the sky plane. We plot the three best-fit orbits in Ginski et al. (2014). We also plot one possible solution from Schwarz et al. (2016), with $a \sim 170$ au and $e \sim 0.25$ constrained by our analysis in Figure 6 . Right: when the disk is viewed edge-on. Two possible disk orientations with identical inclination relative to the line of sight are plotted for comparison. None of these orbits is coplanar with the disk.

representative orbit from Schwarz et al. (2016) for which the semimajor axis and eccentricity are constrained by our tidal truncation analysis in Section 4.3, and other parameters including the longitude of the ascending node and the argument of periastron are extracted from unpublished astrometric fitting (C. Ginski 2016, private communication). All these orbits are unlikely to be in the same plane of the disk. Although A's disk and B's orbit may share similar inclinations, they probably have very different orientations in space.

In Figure 8, we plot a possible geometry of the GQ Lup system. The circumstellar disk is not aligned with the star's spin axis either, because they have different inclinations: $\sim 56^{\circ}$ for the disk and $\sim 27^{\circ}$ for the spin axis (Broeg et al. 2007). This is not unusual among T Tauri stars. Appenzeller \& Bertout (2013) showed that, although stellar rotation angle is correlated with disk inclination, they have a mean difference of $\sim 19^{\circ}$ in T Tauri systems. We note that this misalignment might be induced by a torque from GQ Lup B (e.g., Batygin 2012; Huber et al. 2013).

We caution that the results presented here and in Section 4.3 are preliminary. As Ginski et al. (2014) and Schwarz et al. (2016) stressed, many orbital solutions share similar $\chi^{2}$ in their orbital fitting. Future astrometric monitoring is essential to lift degeneracies and ascertain GQ Lup B's orbit.

\subsection{SED of $G Q$ Lup $B$}

Figure 9 compares the spectral energy distribution (SED) of GQ Lup B to the $2400 \mathrm{~K}, \log g=4.0 \mathrm{BT}$-Settl model (Allard et al. 2011). The temperature and surface gravity are chosen to be consistent with previous estimates (e.g., Marois et al. 2007; McElwain et al. 2007; Lavigne et al. 2009). The model is normalized at $K$ to minimize the effects from dust emission and extinction. We include 0.3 to $3.7 \mu \mathrm{m}$ literature photometry, the $3 \sigma$ upper limit of $0.643 \mu \mathrm{m}$, and fluxes of $\mathrm{H} \alpha, i^{\prime}, z^{\prime}$, and $Y_{S}$ in the figure.

Overall, the $2400 \mathrm{~K}$ model gives a reasonable fit longward of $0.7 \mu \mathrm{m}$. At shorter wavelengths, the observed fluxes are much higher than photospheric due to excess continuum emission from accretion (Zhou et al. 2014). The $0.656 \mu \mathrm{m} \mathrm{H} \alpha$ emission is especially prominent. As recently simulated by Szulágyi \&

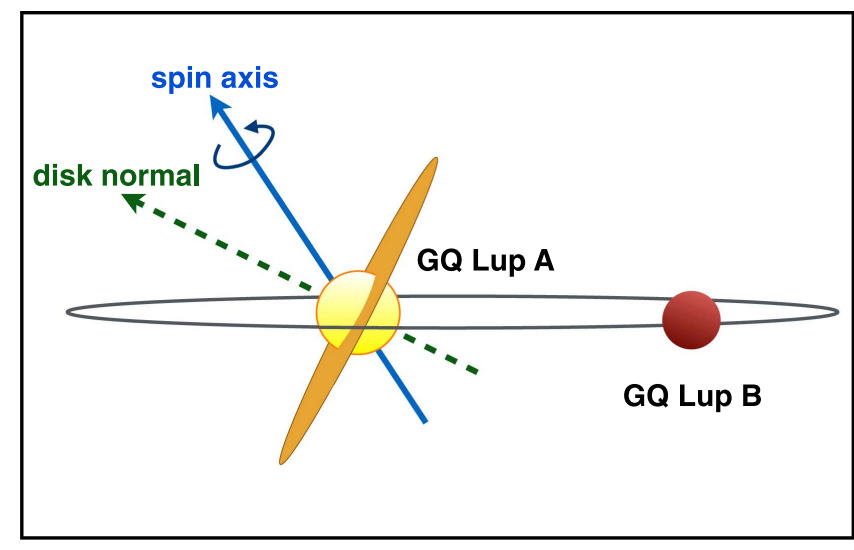

Figure 8. Our rendition of the GQ Lup system. GQ Lup B is perhaps on a low eccentricity orbit not coplanar with the circumstellar disk. In addition, GQ Lup A's spin axis is not aligned with the disk $\left(i \sim 27^{\circ}\right.$ vs. $\left.\sim 56^{\circ}\right)$. Sizes are not to scale.

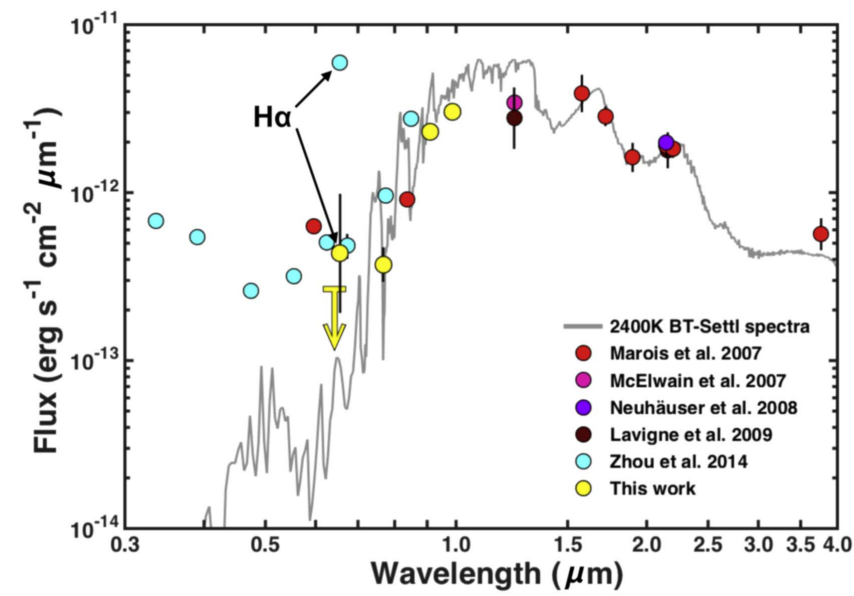

Figure 9. SED of GQ Lup B. We add the observed $R$ variability amplitude of the star (0.7 mag; Broeg et al. 2007) to our $\mathrm{H} \alpha$ flux uncertainty. The $3 \sigma$ limit for $0.643 \mu \mathrm{m}$ also shares that large uncertainty, but it is not shown for clarity. Many data have error bars smaller than the symbols. 
Mordasini (2017), $\mathrm{H} \alpha$ emission likely comes from the extended shock front on the surface of the circumsubstellar disk. Our measured $\mathrm{H} \alpha$ flux, albeit with a large uncertainty, is about 10 times fainter than that of Zhou et al. (2014). Our $0.643 \mu \mathrm{m}$ nondetection also indicates a much weaker accretion continuum. Therefore, accretion onto GQ Lup B seems to be quite variable; we probably observed a more quiescent phase than Zhou et al.

\subsection{Accretion Rate and Disk Lifetime}

Disk lifetime can be roughly estimated assuming a constant accretion rate, but it should be taken with caution because accretion can vary significantly. Our measured $\dot{M}$ for GQ Lup A, $10^{-8}$ to $10^{-7} M_{\odot} \mathrm{yr}^{-1}$, is typical of T Tauri stars (e.g., Natta et al. 2006). With $M_{\text {disk }} \sim 2 \times 10^{-4} M_{\odot}$, GQ Lup A's disk may be depleted in a few thousand years. For the companion, with a disk mass upper limit of $\sim 10^{-5} M_{\odot}$ found by MacGregor et al. (2017), and an accretion rate of $\dot{M} \sim 10^{-12}$ to $5 \times 10^{-10} M_{\odot} \mathrm{yr}^{-1}$ derived by Zhou et al. (2014) and our $\mathrm{H} \alpha$ photometry, GQ Lup B's disk perhaps can continue for tens of thousands of years. The disk lifetime for GQ Lup B might be longer than that of the host star.

\section{Discussion}

\subsection{Formation of GQ Lup B: Scattering}

Debes \& Sigurdsson (2006) argued that scattering might be the most favorable scenario for GQ Lup B, where it originally formed close to the star, but was scattered outward by a more massive body. Searches for a close-in massive companion to the star have not yielded positive results. Deep AO imaging in Neuhäuser et al. (2008) excluded any object as bright as GQ Lup B outside $\sim 18$ au. Similarly, RV monitoring rejected objects more massive than $0.1 M_{\odot}$ within 2.6 au (Broeg et al. 2007), but Donati et al. (2012) speculated that a massive brown dwarf might reside only a few astronomical units from the star in order to explain their observed $0.4 \mathrm{~km} \mathrm{~s}^{-1} \mathrm{RV}$ change in two years. However, if such an inner object exists, morphology of GQ Lup A's disk can be used to infer its presence since it may sculpt a gap or hole. The inner edge of a circumbinary disk can be truncated at approximately two to three times the binary separation (Artymowicz \& Lubow 1994), corresponding to a $\sim 10$ au hole for the brown dwarf companion posited by Donati et al. (2012). Nevertheless, neither the $0.3 \mu \mathrm{m}$ to $1.3 \mathrm{~mm}$ disk SED (McClure et al. 2012) nor our ALMA $1.3 \mathrm{~mm}$ diskresolved map find evidence of a gap or central clearing in the disk. Thus, it is quite unlikely that another massive body is very close to the primary star to serve as the scatterer.

In addition, scattering often induces a very eccentric orbit (e.g., Nagasawa \& Ida 2011), but for GQ Lup B, orbital solutions with low eccentricities are more probable, as shown in Section 4.3. Therefore, all lines of observational evidence suggest that in situ formation via disk fragmentation or prestellar core collapse is more likely the formation pathway of GQ Lup B. This is in line with the null result of the dedicated AO search for scatterers in other systems (Bryan et al. 2016); thus, core accretion plus subsequent scattering is perhaps not responsible for most substellar companions on wide orbits.

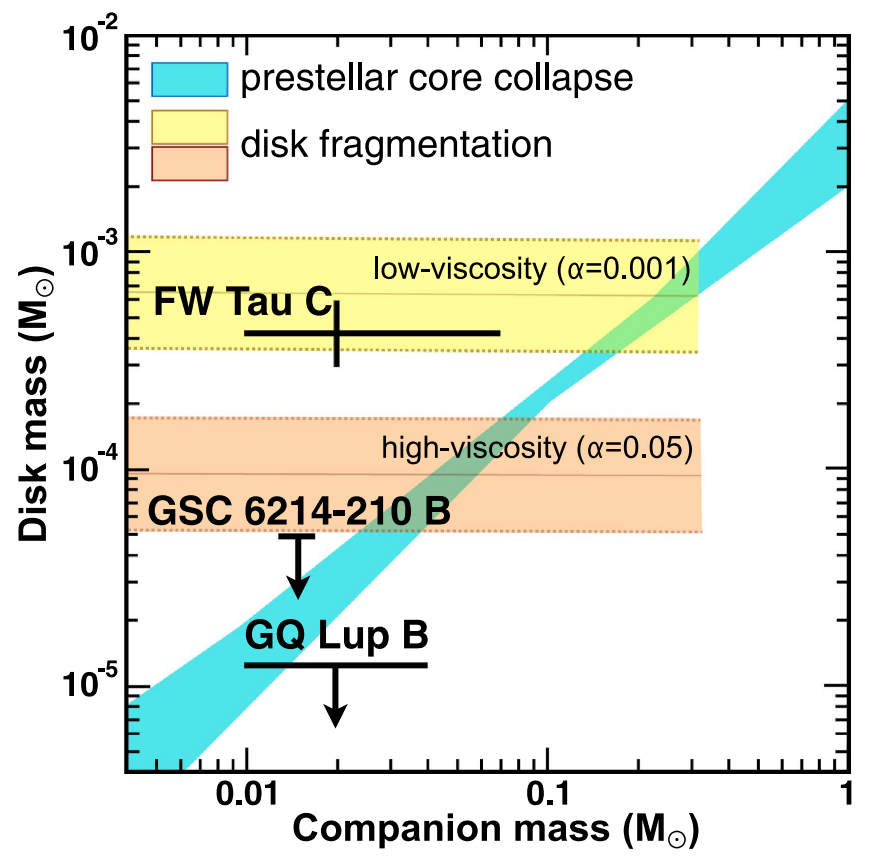

Figure 10. Mass of disk vs. mass of companion for two formation pathways. Figure adapted from Stamatellos \& Herczeg (2015). Turquoise area represents the best fits to the Taurus star-forming region in Andrews et al. (2013), without including the 0.7 dex of standard deviation. Yellow and salmon swaths are the $\pm 1 \sigma$ intervals for 1 to 10 Myr objects formed by disk fragmentation. Disk masses for substellar companions are adopted from Kraus et al. (2015), Bowler et al. (2015), and MacGregor et al. (2017). GQ Lup B seems to be more consistent with the formation via prestellar core collapse.

\subsection{Formation of $G Q$ Lup B: in situ}

Observationally distinguishing prestellar core collapse from disk fragmentation requires high-resolution imaging toward the earliest phase in star formation. Recent studies suggest that prestellar core collapse can be effective to form very wide $(>1000 \mathrm{au})$ binary or multiple star systems (e.g., Pineda et al. 2015), while disk fragmentation may form more compact systems with separations of tens to hundreds of astronomical units between the components (e.g., Tobin et al. 2016). At $\sim 110$ au from the host star, GQ Lup B seems to fit nicely to the disk fragmentation scenario.

For fragmentation to occur, GQ Lup A's disk must have been very massive and presumably more extended than 50 to $100 \mathrm{au}$, since circumstellar disks are expected to become gravitationally unstable beyond that radius (e.g., Clarke 2009). It is sometimes suggested that the disk plane and the companion's orbital plane should be coplanar because the companion formed in the disk. However, dynamical interactions with other fragments in the parent disk can gradually alter the initial configuration, thereby creating inclined systems (Stamatellos \& Whitworth 2009). As a result, even though in Section 4.4 we have shown that GQ Lup B's orbital plane is probably not coplanar with GQ Lup A's disk, disk fragmentation remains a possibility.

Recently, Stamatellos \& Herczeg (2015) proposed that properties of circumsubstellar disks provide an observational diagnostic to distinguish disk fragmentation from prestellar core collapse. They predicted higher disk masses and accretion rates for objects formed via disk fragmentation, because they have a longer time to accrete and thus retain a more massive disk. The discrepancy between the two scenarios is more 
profound for very-low-mass companions, especially $<10 M_{\text {Jup }}$. The authors also predicted that under the disk fragmentation framework low-viscosity circumsubstellar disks tend to have masses and accretion rates higher than that of high-viscosity ones, because higher viscosity facilitates angular momentum transport and disk dissipation.

In Figure 10, we overplot substellar companions FW Tau C, GSC 6214-210 B, and GQ Lup B on the Figure 7 of Stamatellos \& Herczeg (2015). Objects formed by disk fragmentation have a roughly constant disk mass, in drastic contrast to the monotonic correlation $M_{\text {disk }} \propto M_{\text {star }}$ for prestellar core collapse. While FW Tau $\mathrm{C}$ has a rather massive disk, considering the large dispersion in the $M_{\text {disk }} \propto M_{\text {star }}$ correlation ( \pm 0.7 dex; Andrews et al. 2013), it is still consistent with both formation scenarios. The very-low-mass disks around GQ Lup B and GSC 6214-210 B are more in line with the formation via prestellar core collapse. Alternatively, if they formed via disk fragmentation, their disks might have a high viscosity to quickly dissipate.

\subsection{Formation of Satellites}

Disks around wide-orbit substellar companions provide clues to the formation and population of exomoons, which are challenging to detect with current facilities. Simulations of Payne \& Lodato (2007) found that Jupiter-mass satellites are unlikely to form around brown dwarfs because no rocky cores grow fast enough to accrete a gaseous envelope before the disk dissipates, while Earth-like satellites can be common if disk mass is a few $M_{\text {Jup }}$. Nonetheless, even Earth-mass satellites are rare if disk mass is only a fraction of $M_{\text {Jup }}$. Thus, it appears that GQ Lup B has no gaseous moons, while a few Earth-like moons may have formed in early times when the disk was more massive. As MacGregor et al. (2017) and our ALMA observations find that GQ Lup B's disk is deficient in dust, forming Earth-like satellites is no longer possible. Only tiny rocky moons analogous to the Moon $\left(\sim 0.012 M_{\oplus}\right)$ may still form out of the remaining material $\left(<0.04 M_{\oplus}\right)$. Satellite formation around GQ Lup B is probably in the late stage and may have already ceased.

\section{Conclusions}

We observe the 2-5 Myr GQ Lup system with ALMA at $1.3 \mathrm{~mm}$ and MagAO at 0.6 to $1 \mu \mathrm{m}$. With an unprecedented 0 ." $054 \times 0$ ". 031 resolution at $1.3 \mathrm{~mm}$, we resolve GQ Lup A's accretion disk. Our observations, however, are not deep enough to detect GQ Lup B's disk. The main results are as follows.

1. GQ Lup A's disk has a radius of $\sim 22$ au, a dust mass of $\sim 6 M_{\oplus}$, an inclination angle of $\sim 56^{\circ}$, a position angle of $\sim 349^{\circ}$, and a very flat surface density profile. The flat profile is indicative of radial variation of dust sizes, with larger grains growing in the inner disk. This is also supported by the larger disk size measured at a shorter wavelength of $870 \mu \mathrm{m}$ (MacGregor et al. 2016).

2. GQ Lup A's disk is not aligned with the star's spin axis ( $i \sim 56^{\circ}$ versus $\sim 27^{\circ}$ ), and it is unlikely to be coplanar with GQ Lup B's orbit. We use the size of the GQ Lup A disk to demonstrate that GQ Lup B's orbit might have a low eccentricity $e \sim 0.2-0.3$ with a semimajor axis of $a \sim 160-180$ au. Highly eccentric orbits have tidal truncation radii incompatible with the measured disk size.
3. Both components are glowing in $\mathrm{H} \alpha$, indicating active accretion. We derive accretion rates of $\dot{M} \sim 10^{-8}$ to $10^{-7} M_{\odot} \mathrm{yr}^{-1}$ for GQ Lup A, and $\dot{M} \sim 10^{-12}$ to $10^{-11} M_{\odot} \mathrm{yr}^{-1}$ for GQ Lup B. This implies that GQ Lup A's disk may be depleted in a few thousand years, while GQ Lup B's disk may remain longer.

4. Both our disk modeling and the more sensitive observations by MacGregor et al. (2017) suggest that GQ Lup B's disk is rather dust-depleted, similar to GSC 6214-210 B $\left(<0.15 M_{\oplus}\right.$ of dust; Bowler et al. 2015), but in contrast to the dust-abundant disk around FW Tau C (1-2 $M_{\oplus}$ of dust; Kraus et al. 2015). This may be due to age differences because GQ Lup and GSC 6214-210 are old compared with FW Tau.

5. Since there are no gaps and no inner cavity in GQ Lup A's disk, the chance of having another inner companion more massive than GQ Lup B is low. Therefore, it is unlikely that scattering is responsible for GQ Lup B's formation; in situ formation via disk fragmentation or prestellar core collapse is favored. The very-low-mass disk of GQ Lup B is more consistent with prestellar core collapse based on the simulations in Stamatellos \& Herczeg (2015).

6. Based on the results of Payne \& Lodato (2007), GQ Lup $\mathrm{B}$ probably has no gaseous satellites. With very little dust remaining in the disk, only tiny rocky moons might form around GQ Lup B.

We thank the referee for helpful comments. We are grateful to Christian Ginski and Henriette Schwarz for providing their new astrometric fitting, and Yifan Zhou for the $\mathrm{H} \alpha$ contrast in the HST data. We thank Kaitlin Kratter, Min-Kai Lin, Yu-Cian Hong, and Jing-Hua Lin for discussions. We are also grateful to the MagAO development team and the Magellan Observatory staff for their support. This material is based upon work supported by the National Science Foundation under Grant No. 1506818 (PI Males) and NSF AAG Grant No. 1615408 (PI Close). Y.-L.W. and L.M.C. are supported by the NASA Origins of Solar Systems award and the TRIF fellowship. J.R.M. and K. M.M. were supported under contract with the California Institute of Technology (Caltech) funded by NASA through the Sagan Fellowship Program. K.M.M.'s and L.M.C.'s work is supported by the NASA Exoplanets Research Program (XRP) by cooperative agreement NNX16AD44G. This paper makes use of the following ALMA data: ADS/JAO.ALMA\# 2015.1.00773.S. ALMA is a partnership of ESO (representing its member states), NSF (USA) and NINS (Japan), together with NRC (Canada), NSC and ASIAA (Taiwan), and KASI (Republic of Korea), in cooperation with the Republic of Chile. The Joint ALMA Observatory is operated by ESO, AUI/NRAO, and NAOJ. The National Radio Astronomy Observatory is a facility of the National Science Foundation operated under cooperative agreement by Associated Universities, Inc. Results from distributed computing were obtained using the Chameleon testbed supported by the National Science Foundation.

\section{References}

Allard, F., Homeier, D., \& Freytag, B. 2011, in ASP Conf. Ser. 448, XVI Cambridge Workshop on Cool Stars, Stellar Systems, and the Sun, ed. C. Johns-Krull, M. K. Browning, \& A. A. West (San Francisco, CA: ASP), 91

Andrews, S. M., Rosenfeld, K. A., Kraus, A. L., \& Wilner, D. J. 2013, ApJ, 771,129 
Andrews, S. M., Wilner, D. J., Espaillat, C., et al. 2011, ApJ, 732, 42 Appenzeller, I., \& Bertout, C. 2013, A\&A, 558, A83

Appenzeller, I., Mundt, R., \& Wolf, B. 1978, A\&A, 63, 289

Artymowicz, P., \& Lubow, S. H. 1994, ApJ, 421, 651

Ayliffe, B. A., \& Bate, M. R. 2009, MNRAS, 397, 657

Bailey, V., Hinz, P. M., Currie, T., et al. 2013, ApJ, 767, 31

Batalha, C., Lopes, D. F., \& Batalha, N. M. 2001, ApJ, 548, 377

Bate, M. R. 2012, MNRAS, 419, 3115

Batygin, K. 2012, Natur, 491, 418

Beckwith, S. V. W., Sargent, A. I., Chini, R. S., \& Guesten, R. 1990, AJ, 99, 924

Bertout, C., Carrasco, L., Mundt, R., \& Wolf, B. 1982, A\&AS, 47, 419

Bonnefoy, M., Lagrange, A.-M., Boccaletti, A., et al. 2011, A\&A, 528, L15

Bowler, B. P., Andrews, S. M., Kraus, A. L., et al. 2015, ApJL, 805, L17

Bowler, B. P., Liu, M. C., Kraus, A. L., \& Mann, A. W. 2014, ApJ, 784, 65

Bowler, B. P., Liu, M. C., Kraus, A. L., Mann, A. W., \& Ireland, M. J. 2011, ApJ, 743, 148

Broeg, C., Schmidt, T. O. B., Guenther, E., et al. 2007, A\&A, 468, 1039

Bryan, M. L., Bowler, B. P., Knutson, H. A., et al. 2016, ApJ, 827, 100

Caceres, C., Hardy, A., Schreiber, M. R., et al. 2015, ApJL, 806, L22

Clarke, C. J. 2009, MNRAS, 396, 1066

Close, L. M., Follette, K. B., Males, J. R., et al. 2014, ApJL, 781, L30

Close, L. M., Males, J. R., Kopon, D., et al. 2012, Proc. SPIE, 8447, 84470X

Close, L. M., Males, J. R., Morzinski, K., et al. 2013, ApJ, 774, 94

Covino, E., Terranegra, L., Franchini, M., Chavarría-K, C., \& Stalio, R. 1992, A\&AS, 94, 273

Crawford, I. A. 2000, MNRAS, 317, 996

Currie, T., Cloutier, R., Brittain, S., et al. 2015, ApJL, 814, L27

Czekala, I., Andrews, S. M., Jensen, E. L. N., et al. 2015, ApJ, 806, 154

Czekala, I., Andrews, S. M., Torres, G., et al. 2016, ApJ, 818, 156

Dai, Y., Wilner, D. J., Andrews, S. M., \& Ohashi, N. 2010, AJ, 139, 626

Debes, J. H., \& Sigurdsson, S. 2006, A\&A, 451, 351

Donati, J.-F., Gregory, S. G., Alencar, S. H. P., et al. 2012, MNRAS, 425, 2948

Dullemond, C. P. 2012, RADMC-3D: A Multi-purpose Radiative Transfer Tool, Astrophysics Source Code Library, ascl:1202.015

Eggleton, P. P. 1983, ApJ, 268, 368

Foreman-Mackey, D., Hogg, D. W., Lang, D., \& Goodman, J. 2013, PASP, 125,306

Franco, G. A. P. 2002, MNRAS, 331, 474

Friedrich, D., \& Schöffel, E. 1971, IBVS, 558, 1

Ginski, C., Schmidt, T. O. B., Mugrauer, M., et al. 2014, MNRAS, 444, 2280

Haug-Baltzell, A., Males, J. R., Morzinski, K. M., et al. 2016, Proc. SPIE, 9913, 9913

Herbig, G. H. 1962, AdA\&A, 1, 47

Herbig, G. H. 1977, ApJ, 214, 747

Huber, D., Carter, J. A., Barbieri, M., et al. 2013, Sci, 342, 331

Hügelmeyer, S. D., Dreizler, S., Hauschildt, P. H., et al. 2009, A\&A, 498, 793

Hughes, J., Hartigan, P., Krautter, J., \& Kelemen, J. 1994, AJ, 108, 1071

Isella, A., Chandler, C. J., Carpenter, J. M., Pérez, L. M., \& Ricci, L. 2014, ApJ, 788, 129

Janson, M., Brandner, W., Henning, T., \& Zinnecker, H. 2006, A\&A, 453, 609

Johns-Krull, C. M., Chen, W., Valenti, J. A., et al. 2013, ApJ, 765, 11

Kardopolov, V. I., \& Filipev, G. K. 1985, PZ, 22, 103

Kessler-Silacci, J., Augereau, J.-C., Dullemond, C. P., et al. 2006, ApJ, 639, 275

Kopon, D., Close, L. M., Males, J. R., \& Gasho, V. 2013, PASP, 125, 966

Kraus, A. L., Andrews, S. M., Bowler, B. P., et al. 2015, ApJL, 798, L23

Kraus, A. L., Ireland, M. J., Cieza, L. A., et al. 2014, ApJ, 781, 20

Lachapelle, F.-R., Lafrenière, D., Gagné, J., et al. 2015, ApJ, 802, 61
Lavigne, J.-F., Doyon, R., Lafrenière, D., Marois, C., \& Barman, T. 2009, ApJ, 704, 1098

Lynden-Bell, D., \& Pringle, J. E. 1974, MNRAS, 168, 603

MacGregor, M. A., Wilner, D. J., Czekala, I., et al. 2017, ApJ, 835, 17

Males, J. R. 2016, MAOP-710: VisAO Photometric Calibration (Tucson, AZ: Univ. Arizona Press) https://visao.as.arizona.edu/wp-content/uploads 2016/09/nd_cal_2016.09_21.pdf

Males, J. R., Close, L. M., Morzinski, K., et al. 2014, ApJ, 786, 32

Marois, C., Lafrenière, D., Doyon, R., Macintosh, B., \& Nadeau, D. 2006, ApJ, 641,556

Marois, C., Macintosh, B., \& Barman, T. 2007, ApJL, 654, L151

McClure, M. K., Manoj, P., Calvet, N., et al. 2012, ApJL, 759, L10

McElwain, M. W., Metchev, S. A., Larkin, J. E., et al. 2007, ApJ, 656, 505

Mendoza, E. E. 1968, ApJ, 151, 977

Morales, F. Y., Padgett, D. L., Bryden, G., Werner, M. W., \& Furlan, E. 2012, ApJ, 757, 7

Morzinski, K. M., Close, L. M., Males, J. R., et al. 2014, Proc. SPIE, 9148 914804

Morzinski, K. M., Males, J. R., Skemer, A. J., et al. 2015, ApJ, 815, 108

Mugrauer, M., \& Neuhäuser, R. 2005, AN, 326, 701

Nagasawa, M., \& Ida, S. 2011, ApJ, 742, 72

Natta, A., Testi, L., \& Randich, S. 2006, A\&A, 452, 245

Neuhäuser, R., Guenther, E. W., Wuchterl, G., et al. 2005, A\&A, 435, L13

Neuhäuser, R., Mugrauer, M., Seifahrt, A., Schmidt, T. O. B., \& Vogt, N. 2008, A\&A, 484, 281

Patience, J., King, R. R., De Rosa, R. J., et al. 2012, A\&A, 540, A85

Payne, M. J., \& Lodato, G. 2007, MNRAS, 381, 1597

Pichardo, B., Sparke, L. S., \& Aguilar, L. A. 2005, MNRAS, 359, 521

Pineda, J. E., Offner, S. S. R., Parker, R. J., et al. 2015, Natur, 518, 213

Quillen, A. C., \& Trilling, D. E. 1998, ApJ, 508, 707

Rau, U., \& Cornwell, T. J. 2011, A\&A, 532, A71

Reipurth, B., \& Clarke, C. 2001, AJ, 122, 432

Ricci, L., Testi, L., Natta, A., et al. 2014, ApJ, 791, 20

Rigliaco, E., Natta, A., Testi, L., et al. 2012, A\&A, 548, A56

Sallum, S., Follette, K. B., Eisner, J. A., et al. 2015, Natur, 527, 342

Schmidt, T. O. B., Neuhäuser, R., Seifahrt, A., et al. 2008, A\&A, 491, 311

Schwartz, R. D., \& Noah, P. 1978, AJ, 83, 785

Schwarz, H., Ginski, C., de Kok, R. J., et al. 2016, A\&A, 593, 74

Seifahrt, A., Neuhäuser, R., \& Hauschildt, P. H. 2007, A\&A, 463, 309

Seperuelo Duarte, E., Alencar, S. H. P., Batalha, C., \& Lopes, D. 2008, A\&A, 489, 349

Shabram, M., \& Boley, A. C. 2013, ApJ, 767, 63

Soummer, R., Pueyo, L., \& Larkin, J. 2012, ApJL, 755, L28

Stamatellos, D., \& Herczeg, G. J. 2015, MNRAS, 449, 3432

Stamatellos, D., \& Whitworth, A. P. , 2009, MNRAS, 392, 413

Szulágyi, J., \& Mordasini, C. 2017, MNRAS, 465, L64

Testi, L., Natta, A., Scholz, A., et al. 2016, A\&A, 593, 111

Tobin, J. J., Kratter, K. M., Persson, M. V., et al. 2016, Natur, 538, 483

Tody, D. 1986, Proc. SPIE, 627, 733

Tody, D. 1993, in ASP Conf. Ser. 52, Astronomical Data Analysis Software and Systems II, ed. R. J. Hanisch, R. J. V. Brissenden, \& J. Barnes (San Francisco, CA: ASP), 173

Uyama, T., Hashimoto, J., Kuzuhara, M., et al. 2017, AJ, 153, 106

Weingartner, J. C., \& Draine, B. T. 2001, ApJ, 548, 296

White, R. J., \& Ghez, A. M. 2001, ApJ, 556, 265

Wu, Y.-L., Close, L. M., Males, J. R., et al. 2015a, ApJ, 801, 4

Wu, Y.-L., Close, L. M., Males, J. R., et al. 2015b, ApJL, 807, L13

Zacharias, N., Finch, C., Girard, T., et al. 2010, AJ, 139, 2184

Zhou, Y., Herczeg, G. J., Kraus, A. L., Metchev, S., \& Cruz, K. L. 2014, ApJL, 783, L17 